\title{
Collision of particles near a three-dimensional rotating Hořava AdS black hole
}

\author{
Ramón Bécar ${ }^{1, a}$, P. A. González ${ }^{2, b}$, Yerko Vásquez ${ }^{3, c}$ \\ ${ }^{1}$ Departamento de Ciencias Matemáticas y Físicas, Universidad Católica de Temuco, Montt 56, Casilla 15-D, Temuco, Chile \\ ${ }^{2}$ Facultad de Ingeniería y Ciencias, Universidad Diego Portales, Avenida Ejército Libertador 441, Casilla 298-V, Santiago, Chile \\ ${ }^{3}$ Departamento de Física, Facultad de Ciencias, Universidad de La Serena, Avenida Cisternas 1200, La Serena, Chile
}

Received: 19 February 2020 / Accepted: 8 March 2021 / Published online: 22 March 2021

(C) The Author(s) 2021

\begin{abstract}
We consider a three-dimensional rotating AdS black hole, which is a solution of Hořava gravity in the lowenergy limit that corresponds to a Lorentz-violating version of the BTZ black hole, and we analyze the effect of the breaking of Lorentz invariance on the possibility that the black hole can act as a particle accelerator by analyzing the energy in the center-of-mass (CM) frame of two colliding particles in the vicinity of its horizons. We find that the critical angular momentum of particles increases when the Hořava parameter $\xi$ increases and when the aether parameter $b$ increases. Also, the particles can collide on the inner horizon with arbitrarily high CM energy if one of the particles has a critical angular momentum, possible for the BSW process. Here it is essential that, while for the extremal BTZ black hole the particles with critical angular momentum only can exist on the degenerate horizon, for the Lorentz-violating version of the BTZ black hole the particle with critical angular momentum can exist in a region away from the degenerate horizon. It is worth mentioning that the results exposed in this manuscript can be applied for the covariant version of Hořava gravity, where the covariant definition of the center-of-mass energy is well defined.
\end{abstract}

\section{Contents}

1 Introduction .................. 1

2 Three-dimensional Einstein-Aether black holes and Hořava gravity equivalence . . . . . . . . . . 2

3 Equations of motion . . . . . . . . . . . 4

4 The CM energy of two colliding particles ..... 5

\footnotetext{
a e-mail: rbecar@uct.cl

be-mail: pablo.gonzalez@udp.cl

c e-mail: yvasquez@userena.cl (corresponding author)
}

5 Radial motion of the particle with critical angular momentum . . . . . . . . . . 7

6 Final remarks . . . . . . . . . . . . . . . 8

References . . . . . . . . . . . . . . 10

\section{Introduction}

Bañados, Silk and West (BSW) [1] demonstrated that two particles colliding near the degenerate horizon of an extreme Kerr black hole could create a large center-of-mass (CM) energy if one of the particles has a critical angular momentum; thus, extreme Kerr black holes can act as natural particle accelerators. Nowadays, this process is known as the BSW mechanism; it was found for the first time by Piran, Shaham and Katz in 1975 [2-4]. The same mechanism also occurs for non-extremal ones [5], and it is a universal property of rotating black holes [6]. Also, it was shown that a similar effect exists for non-rotating charged black holes [7]. Moreover, the extension of the BSW mechanism to non-extremal backgrounds shows that particles cannot collide with arbitrarily high energies at the outer horizon and that ultra-energetic collisions can only occur near the Cauchy horizon of a Kerr black hole with any spin parameter [8]. Non-extremal Kerr-de Sitter black holes could also act as particle accelerators with arbitrarily high CM energy if one of the colliding particles has the critical angular momentum [9]. The BSW mechanism has been extensively studied for different black hole geometries [10-30]. Furthermore, the formation of black holes through the BSW mechanism was investigated in [31].

Nowadays, one could think that Lorentz invariance may not be fundamental or exact, but is merely an emergent symmetry on sufficiently large distances or low energies. It has been suggested in Ref. [32] that giving up Lorentz invariance by introducing a preferred foliation and terms that contain higher-order spatial derivatives can lead to significantly 
improved UV behavior, and the corresponding gravity theory is dubbed Hořava gravity. On the other hand, there was proposed a Lorentz-violating theory of gravity with an aether vector field $u_{\mu}$, determining a preferred rest frame at each spacetime point; the so-called Einstein-Aether (EA) gravity theory [33]. More precisely, $u_{\mu}$ breaks local boost invariance, while rotational symmetry in a preferred frame is preserved. Because Hořava and the EA theories are both modifications of gravity which break Lorentz symmetry, maybe the two are related. In fact, in the limit where higher than second order derivative terms of the Hořava theory can be ignored (which corresponds to the IR limit of the theory), one obtains the EA theory with the additional constraint that the aether vector should be hypersurface orthogonal. Then one can express the aether in terms of a scalar $T$ as

$u_{\mu}=\frac{\partial_{\mu} T}{\sqrt{g^{\mu \nu} \partial_{\mu} T \partial_{\nu} T}}$.

The arguments given suggest that the $E A$ theory can be seen dynamically as a covariant version of the low-energy regime of Horăva gravity. The three-dimensional Hořava gravity [34] admits a Lorentz-violating version of the BTZ black hole, i.e. a black hole solution with AdS asymptotics, only in the sector of the theory in which the scalar degree of freedom propagates infinitely fast [35]. Remarkably, in contrast to general relativity, the three-dimensional Hořava gravity also admits black holes with positive and vanishing cosmological constant. The propagation of a massive scalar field is stable in this background [36]. Also, new kinds of orbits are allowed, such as unstable circular orbits and trajectories of the first kind for the motion of photons [37], and particles [38]. The aim of this work is to consider three-dimensional rotating Hořava AdS black holes [35] and to study, via the BSW mechanism, the possibility of obtaining an unbounded energy in the $\mathrm{CM}$ frame of two colliding particles and to analyze the effect of a Lorentz breaking symmetry on this. The BSW mechanism in the case of the Hořava-Lifshitz black hole was studied in four spacetime dimensions $[40,41]$ and in two spacetime dimensions [42]. It is worth mentioning that for extreme rotating black holes in four-dimensional Hořava gravity the fundamental parameter of Hořava gravity can avoid an infinite value of the CM energy [40]. On the other hand particle motions on EA black holes have been studied very recently in $[43,44]$.

In three spacetime dimensions the collision of two particles near the horizon of a BTZ black hole was studied in Refs. [45-47]. In Refs. [45,46] the authors found that the particle with the critical angular momentum could exist inside the outer horizon of the BTZ black hole regardless of the particle energy with the BSW process being possible on the inner horizon for the non-extremal BTZ black hole. Also, the BSW process could also happen for the extremal BTZ black hole, where the particle with the critical angular momentum could only exist on the degenerate horizon. On the other hand, in Ref. [47], the authors studied the collision of two particles on the event horizon and outside of the BTZ black hole, and they showed that although in principle the CM energy of two ingoing particles can be arbitrarily large on the event horizon, if either of the two particles has a critical angular momentum and the other has a non-critical angular momentum, the critical particles never reach the event horizon. However, the motion of a particle with a subcritical angular momentum is allowed for near an extremal rotating BTZ black hole and the CM energy for a tail-on collision at a point can be arbitrarily large in a critical angular momentum limit. Also, the BSW effect is possible on the outer horizon in the extremal warped $\mathrm{AdS}_{3}$ black hole, and the particle with critical angular momentum can reach the degenerate horizon when a condition on its energy is fulfilled [48], which resembles what occurs in the extremal Kerr-AdS black hole; however, in the extremal Kerr-AdS black hole two conditions must be fulfilled [9]; besides, this effect is also possible on the inner horizon for the non-extremal warped $\mathrm{AdS}_{3}$ black hole.

The manuscript is organized as follows: in Sect. 2 we give a brief review of three-dimensional EA black holes and its equivalence with the three-dimensional rotating Hořava black holes in the low-energy limit. Then, in Sect. 3, we study the motion of particles in this background. In Sect. 4, we obtain the CM energy of two colliding particles, and in Sect. 5 we study the radial motion of a particle with critical angular momentum and we investigate the possibility that the black hole acts as a particle accelerator. Finally, our conclusions are in Sect. 6.

\section{Three-dimensional Einstein-Aether black holes and Hořava gravity equivalence}

The $(2+1)$-dimensional EA theory with a cosmological constant $\Lambda$ is described by the action

$S_{\mathfrak{x}}=\frac{1}{16 \pi G_{\mathfrak{x}}} \int \mathrm{d}^{3} x \sqrt{-g}\left(-R-2 \Lambda+L_{\mathfrak{x}}\right)$,

where $R$ is the Ricci scalar, $g$ is the determinant of the metric and

$L_{\mathfrak{x}}=-M_{\mu \nu}^{\alpha \beta} \nabla_{\alpha} u^{\mu} \nabla_{\beta} u^{\nu}$,

where the time vector $u^{\mu}$ satisfies the unit constraint $u^{\alpha} u_{\alpha}=$ 1. $M_{\mu \nu}^{\alpha \beta}$ is defined by

$M^{\alpha \beta \mu \nu}=c_{1} g^{\alpha \beta} g^{\mu \nu}+c_{2} g^{\alpha \mu} g^{\beta \nu}+c_{3} g^{\alpha \nu} g^{\beta \mu}+c_{4} u^{\alpha} u^{\beta} g^{\mu \nu}$. 
The $c_{i}$ are dimensionless coupling constants. Now, by imposing the requirement that the aether is hypersurface orthogonal, locally, this implies that there is a function $T$ for which

$u_{\alpha}=\frac{\partial_{\alpha} T}{\sqrt{g^{\mu \nu} \partial_{\mu} T \partial_{\nu} T}}$,

and, working in a gauge where $T$ is identified with the time coordinate $t$, we have

$u_{\mu}=N \delta_{\mu t}$

So, by replacing Eq. (6) in the action (2), the low-energy limit of the action of Hořava gravity in $2+1$ dimensions is recovered, that is, the action in a preferred foliation is given by [34]

$S_{H}=\frac{1}{16 \pi G_{H}} \int \mathrm{d} T \mathrm{~d}^{2} x N \sqrt{g}\left[\mathcal{L}_{2}+\mathcal{L}_{4}\right]$,

where the Lagrangian $\mathcal{L}_{2}$ is given by

$\mathcal{L}_{2}=K_{i j} K^{i j}-\lambda K^{2}+\xi\left({ }^{(2)} R-2 \Lambda\right)+\eta a_{i} a^{i}$,

where $K_{i j}, K$, and ${ }^{(2)} R$ correspond to extrinsic, mean, and scalar curvature, respectively, and $a_{i}$ is a parameter related to the lapse function $N$ via $a_{i}=-\partial_{i} \ln N$ and without the higher-order operators $\mathcal{L}_{4}$, which corresponds to the set of all the terms with four spatial derivatives that are invariant under diffeomorphisms. The parameters of the two theories are related by the following correspondence:

$\frac{G_{H}}{G_{\mathfrak{x}}}=\xi=\frac{1}{1-c_{13}}, \frac{\lambda}{\xi}=1+c_{2}, \frac{\eta}{\xi}=c_{14}$,

where $G_{H}$ is a coupling constant with the dimensions of length squared. The above arguments suggest that the EA theory can be seen as a covariant version of the IR limit of Hořava gravity. In the covariant formulation of the theory the preferred time $T$ becomes a scalar field that defines the preferred foliation at the level of the solution. The line element in the preferred foliation is written as

$\mathrm{d} s^{2}=N^{2} \mathrm{~d} T^{2}-g_{i j}\left(\mathrm{~d} x^{i}+N^{i} \mathrm{~d} T\right)\left(\mathrm{d} x^{j}+N^{j} \mathrm{~d} T\right)$,

where $g_{i j}$ is the induced metric on the constant- $T$ hypersurfaces, and $g$ is the determinant of $g_{i j}$. Another important characteristic of this theory is that only in the sector $\eta=0$, Hořava gravity admits asymptotically AdS solutions [35]. Therefore, assuming stationary and circular symmetry the most general metric is given by

$$
\mathrm{d} s^{2}=Z(r)^{2} \mathrm{~d} t^{2}-\frac{1}{F(r)^{2}} \mathrm{~d} r^{2}-r^{2}(\mathrm{~d} \phi+\Omega(r) \mathrm{d} t)^{2},
$$

and, by assuming the aether to be hypersurface orthogonal, as a result

$u_{t}= \pm \sqrt{Z(r)^{2}\left(1+F^{2}(r) U^{2}(r)\right)}, u_{r}=U(r)$.

The theory admits the BTZ "analogue" to the three-dimensional rotating Hořava black holes described by the solution

$$
\begin{aligned}
F(r)^{2} & =Z(r)^{2}=-M+\frac{\bar{J}^{2}}{4 r^{2}}-\bar{\Lambda} r^{2}, \Omega(r)=-\frac{J}{2 r^{2}}, \\
U(r) & =\frac{1}{F(r)}\left(\frac{a}{r}+b r\right),
\end{aligned}
$$

where

$\bar{J}^{2}=\frac{J^{2}+4 a^{2}(1-\xi)}{\xi}, \bar{\Lambda}=\Lambda-\frac{b^{2}(2 \lambda-\xi-1)}{\xi}$.

The sign of the effective cosmological constant $\bar{\Lambda}$ determines the asymptotic behavior (flat, dS, or AdS) of the metric. Also, $\bar{J}^{2}$ can be negative; this occurs when either $\xi<0$ or $\xi>1$, $a^{2}>J^{2} /(4(\xi-1))$. The aether configuration for this metric is given explicitly by

$u_{t}=\sqrt{F^{2}+\left(\frac{a}{r}+b r\right)^{2}}, u_{r}=\frac{1}{F^{2}}\left(\frac{a}{r}+b r\right), u_{\phi}=0$,

where $a$ and $b$ are constants that can be regarded as measures of aether misalignment, with $b$ as a measure of the asymptotical misalignment, such that for $b \neq 0$ the aether does not align with the timelike Killing vector asymptotically. Note that, for $\xi=1$ and $\lambda=1$, the solution becomes the BTZ black hole and, for $\xi=1$ and $\lambda \neq 1$, the solution becomes the BTZ black hole with a shifted cosmological constant $\bar{\Lambda}=\Lambda-2 b^{2}(\lambda-1)$. However, there is still a preferred direction represented by the aether vector field which breaks Lorentz invariance for $\lambda \neq 1$ and $b \neq 0$. The locations of the inner and outer horizons, $r=r_{ \pm}$, are given by

$$
r_{ \pm}^{2}=-\frac{M}{2 \bar{\Lambda}}\left(1 \pm \sqrt{1+\frac{\bar{J}^{2} \bar{\Lambda}}{M^{2}}}\right)
$$

and the Hawking temperature $T_{H}$ is given by $T_{H}=$ $\frac{-\bar{\Lambda}\left(r_{+}^{2}-r_{-}^{2}\right)}{2 \pi r_{+}}$. For $\bar{J} \neq J(\xi \neq 1)$, there is a curvature singularity due to the Ricci scalar $R=-6 \bar{\Lambda}+\frac{1}{2 r^{2}}\left(\bar{J}^{2}-J^{2}\right)$, which is divergent at $r=0$, in contrast to the BTZ black holes where the Ricci and Kretschmann scalars are finite and smooth at $r=0$. Considering $M>0$ and a negative cosmological constant $\bar{\Lambda}<0$, the condition $\bar{J}^{2}|\bar{\Lambda}| \leq M^{2}$ must be fulfilled for the solution to represent a black hole. For $0<\bar{J}^{2}|\bar{\Lambda}|<M^{2}$ the black holes have inner and outer horizons $r_{-}$and $r_{+}$, the extremal case corresponds to $\bar{J}^{2}|\bar{\Lambda}|=M^{2}$, while for $\bar{J}^{2}<0$ 


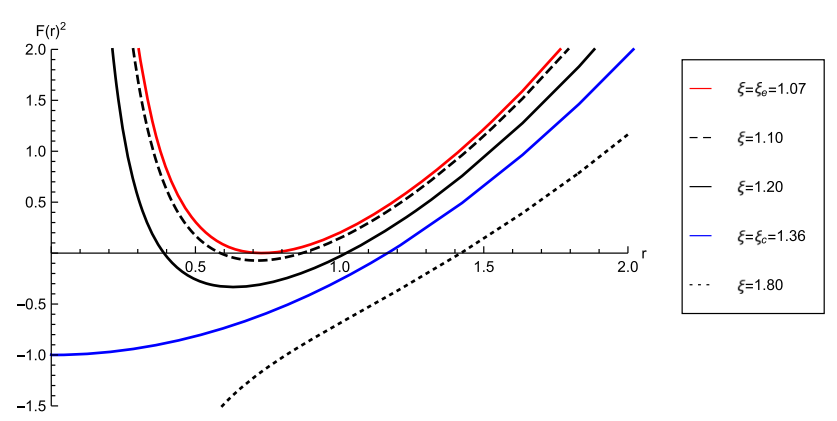

Fig. 1 The behavior of the lapse function as a function of $r$, with $M=1, \lambda=1, a=1, b=1, \Lambda=-1, J=1.2$, for different values of $\xi$

the black holes have an outer horizon $r_{+}$, but no inner horizon $r_{-}$. The value of $\xi$ for which the black hole is extremal is given by [36]

$$
\begin{aligned}
\xi_{e}= & -\frac{1}{2\left(M^{2}-4 a^{2}\left(b^{2}+\Lambda\right)\right)}\left(b^{2}\left(J^{2}+8 a^{2} \lambda\right)+\Lambda\left(J^{2}+4 a^{2}\right)\right. \\
& -\left(\left(b^{2}\left(J^{2}+8 a^{2} \lambda\right)+\Lambda\left(J^{2}+4 a^{2}\right)\right)^{2}\right. \\
& \left.\left.+4 b^{2}\left(J^{2}+4 a^{2}\right)(2 \lambda-1)\left(M^{2}-4 a^{2}\left(b^{2}+\Lambda\right)\right)\right)^{1 / 2}\right)
\end{aligned}
$$

where the subscript $e$ refers to the extremal parameter, that is, the value of $\xi$ such that the two horizons (inner and outer) coalesce. The value of $\xi$ for which the black hole turns from having two horizons to having one horizon is given by $\xi_{c}=\frac{4 a^{2}+J^{2}}{4 a^{2}}$, and the value of $\xi$ for which the effective cosmological constant $\bar{\Lambda}$ changes sign is $\xi=\frac{(2 \lambda-1) b^{2}}{b^{2}+\lambda}$. In Fig. 1, we show the behavior of the lapse function $F(r)^{2}$, for a choice of parameters, and different values of $\xi$, where we can observe the existence of one horizon for $\xi>\xi_{c}(\xi=1.80)$ with $\bar{J}^{2}<0$, one horizon with $r_{-}=0$, for $\xi=\xi_{c} \approx 1.36$ or $\bar{J}=0$, two horizons for $\xi_{e}<\xi<\xi_{c}$, and one degenerate horizon for $\xi=\xi_{e} \approx 1.07$.

Besides the existence of inner and outer horizons, what is perhaps the most interesting feature within the context of Lorentz-violating gravity theories is that they can have universal horizons, which are given by [35]

$$
\begin{aligned}
\left(r_{u}^{ \pm}\right)^{2}= & \frac{M-2 a b}{2\left(b^{2}-\bar{\Lambda}\right)} \pm \frac{1}{2\left(b^{2}-\lambda\right)} \\
& \times\left[(M-2 a b)^{2}-\left(4 a^{2}+\bar{J}^{2}\right)\left(b^{2}-\bar{\Lambda}\right)\right]^{\frac{1}{2}} .
\end{aligned}
$$

In Fig. 2, we plot the behavior of the horizons as a function of the parameter $b$, and as a function of $a$ in Fig. 3, for a choice of parameters. One observes different zones. One of them is limited by $r_{-}$and $r_{+}$; and it is described by the existence of the aether, where the roots $r_{u}^{ \pm}$are imaginary, and therefore there are no universal horizons. Other zones are characterized by two real and distinct universal horizons inside the region between $r_{-}$and $r_{+}$, outside $r_{-}$, and inside $r_{+}$; and a particular

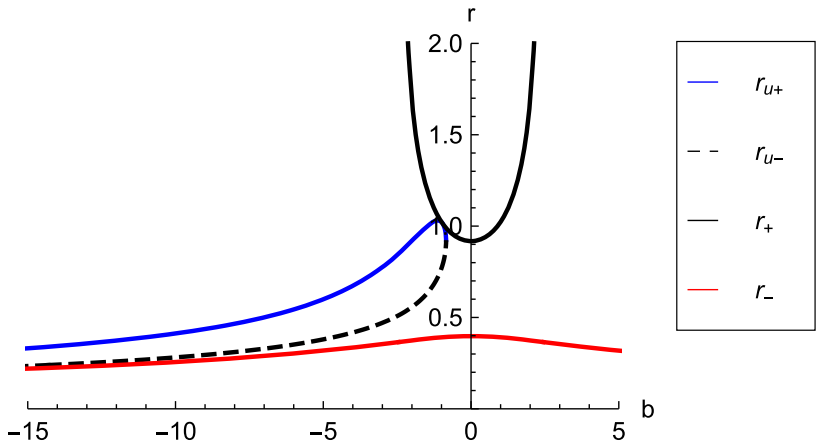

Fig. 2 The behavior of the horizons as a function of the parameter $b$, with $M=1, \xi=1.2, \lambda=1, a=1, \Lambda=-1$, and $J=1.2$. We set $b \approx-0.84, r_{u}^{+}=r_{u}^{-}$

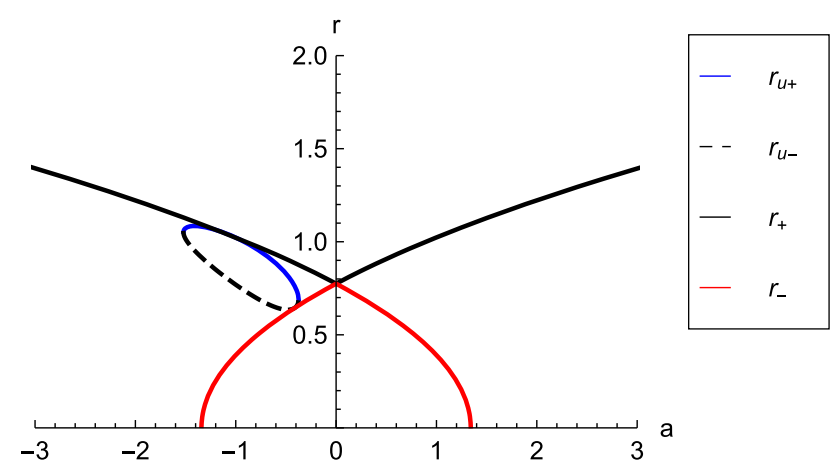

Fig. 3 The behavior of the horizons as a function of the parameter $a$, with $M=1, \xi=1.2, \lambda=1, b=1, \Lambda=-1$, and $J=1.2$. We set $a \approx-1.52$, and $-0.37, r_{u}^{+}=r_{u}^{-}$

zone where the two universal horizons coincide and which is given by

$r_{u}^{2}=\frac{M-2 a_{ \pm}(M, \bar{J}, b) b}{2\left(b^{2}-\bar{\Lambda}(b)\right)}$,

where $a_{ \pm}$are the roots of

$\frac{\left(4 a^{2}+\bar{J}^{2}\right)\left(b^{2}-\bar{\Lambda}(b)\right)}{\xi(M-2 a b)^{2}}=1$.

In the region between $r_{u}^{-}$and $r_{u}^{+}$, the aether turns imaginary and the foliation cannot be extended until the singularity. So, if this region is located between the inner and outer horizon then the BSW process is not possible on the inner horizon $r_{-}$ because the aether is imaginary.

\section{Equations of motion}

In this section, we find the equations of motion of massive probe particles around the three-dimensional Horava AdS black hole. It is important to emphasize [49] that in a Lorentzviolating scenario, particles will be generically coupled to 
the aether field generating UV modifications of the matter dispersion relations; moreover, one might also expect radiative corrections in the infrared sector, but these contributions are suppressed by well-known mechanisms. In our analysis we are interested in the infrared limit of the theory; so the presence of higher-order terms $\left(L_{4}\right)$ related to the UV behavior of the theory is ignored and in this case the theory can be formulated in a covariant fashion, and it then becomes equivalent to a restricted version of EA theory [35]. Since our analysis is focused on the low-energy part of the theory, the interaction between the massive particle and the aether field is ignored, thus the presence of aether field only affects the background spacetime geometry. It is worth mentioning that a similar analysis was performed in [39], where the authors analyzed the evolution of the photon around the static neutral and charged aether black holes using the HamiltonJacobi equation. Therefore, the massive particles follow the typical geodesics in such black holes spacetime, which can be derived from the Lagrangian of a test particle, which is given by [50]

$\mathcal{L}=\frac{1}{2} g_{\mu \nu} \dot{x}^{\mu} \dot{x}^{\nu}$

where $\dot{x}^{\mu}=\mathrm{d} x^{\mu} / \mathrm{d} \tau$, and $\tau$ is an affine parameter along the geodesic that we choose as the proper time. So, for the three-dimensional rotating Hořava AdS black hole described by the metric (11), the Lagrangian reads

$2 \mathcal{L}=\left(Z^{2}(r)-r^{2} \Omega^{2}(r)\right) \dot{t}^{2}-\frac{1}{Z^{2}(r)} \dot{r}-r^{2} \dot{\phi}^{2}-2 r^{2} \Omega(r) \dot{t} \dot{\phi}$.

Since this Lagrangian is independent of the cyclic coordinates $(t, \phi)$, their conjugate momenta $\left(\Pi_{t}, \Pi_{\phi}\right)$ are conserved. The equations of motion are obtained from $\dot{\Pi}_{q}-$ $\frac{\partial \mathcal{L}}{\partial q}=0$, where $\Pi_{q}=\partial \mathcal{L} / \partial \dot{q}$ are the conjugate momenta to the coordinate $q$, given by

$$
\begin{aligned}
\Pi_{t}= & \left(Z^{2}(r)-r^{2} \Omega^{2}(r)\right) \dot{t}+-r^{2} \Omega(r) \dot{\phi} \equiv E, \Pi_{r}=\frac{1}{Z^{2}(r)} \dot{r}, \\
& \Pi_{\phi}=r^{2} \dot{\phi}-r^{2} \Omega(r) \dot{t} \equiv-L,
\end{aligned}
$$

where $E$ and $L$ are dimensionless integration constants associated with each of them. The Hamiltonian

$\mathcal{H}=\Pi_{t} \dot{t}+\Pi_{\phi} \dot{\phi}+\Pi_{r} \dot{r}-\mathcal{L}$

yields

$2 \mathcal{H}=-E \dot{t}+L \dot{\phi}-\frac{\dot{r}^{2}}{Z^{2}(r)} \equiv m^{2}$.
Now, we solve the above equations for $\dot{r}^{2}$ in order to obtain the radial equation which allows us to characterize the possible movements of the test particles without an explicit solution of the equations of motion,

$$
\begin{aligned}
\dot{t} & =\frac{E+L \Omega(r)}{Z^{2}(r)}, \\
\dot{\phi} & =-\frac{E \Omega(r)}{Z^{2}(r)}+L\left(\frac{-\Omega(r)^{2}}{Z^{2}(r)}+\frac{1}{r^{2}}\right), \\
\dot{r}^{2} & =Z^{2}(r)\left(\frac{1}{Z^{2}(r)}(E+L \Omega(r))^{2}-\frac{L^{2}}{r^{2}}-m^{2}\right) .
\end{aligned}
$$

The above equations represent all nonzero 3-velocity components $u=(\dot{t}, \dot{r}, \dot{\phi})$ for the geodesic motion that will be used in the next section to obtain the CM energy of two colliding particles falling freely from rest with the same rest mass $m_{0}$ in the three-dimensional rotating Hořava AdS black hole background. We will assume $\dot{t}>0$ for all $r>r_{+}$, so that the motion is forward in time outside the horizon. So, the following condition must be fulfilled:

$E+L \Omega(r)>0$, for all $r>r_{+}$.

Now, we write the equation of motion of the particle in the radial direction as $\dot{r}^{2}+V(r)=0$, where $V$ is the effective potential of the particle in the radial direction, reading

$V(r)=-\left((E+L \Omega(r))^{2}-Z^{2}(r)\left(\frac{L^{2}}{r^{2}}+m^{2}\right)\right)$.

By analyzing this effective potential we can determine if a particle can reach the event horizon. The motion of the particle is allowed in regions where $V(r) \leq 0$, and it is prohibited in regions where $V(r)>0$. It is clear that the particle can exist on the event horizon $r=r_{+}$because $Z^{2}\left(r_{+}\right)=0$, and then the effective potential is negative. On the other hand, when $r \rightarrow \infty$ it is easy to show that the effective potential approximates

$V(r \rightarrow \infty) \approx-m^{2} \bar{\Lambda} r^{2}$

This expression shows that the existence of a massive particle at infinity depends on the effective cosmological constant $(\bar{\Lambda})$ and not on its energy $E$. Therefore, massive particles cannot exist at infinity in the $A d S$ case, as is shown in Fig. 4 for different values of the parameter $\xi$, and in Fig. 5 for the case $r_{u}^{-}=r_{u}^{+}$.

\section{The CM energy of two colliding particles}

In order to calculate the CM energy of two colliding particles, we use the expressions of the components of the 3velocity derived in the last section to obtain the CM energy 

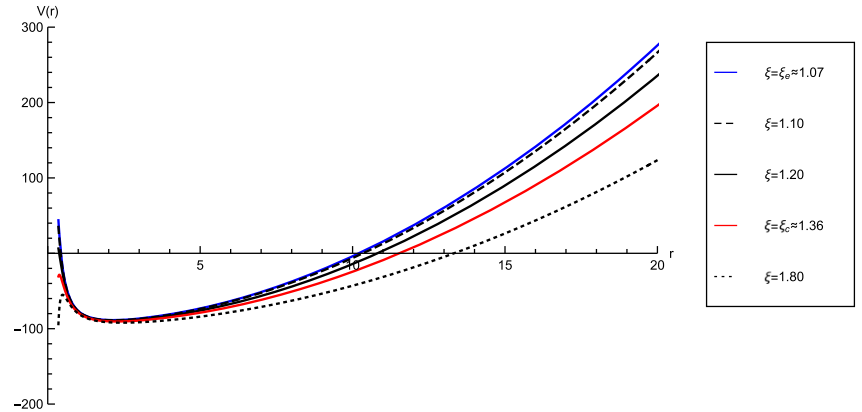

Fig. 4 The behavior of the effective potential $V(r)$ for massive particles $m=1$ as a function of $r$ for different values of $\xi$ and $M=1$, $\lambda=a=b=1, \Lambda=-1, J=1.2, E=10$. The left figure shows the behavior of the effective potential for $L=2$, and $r>0.35$, where

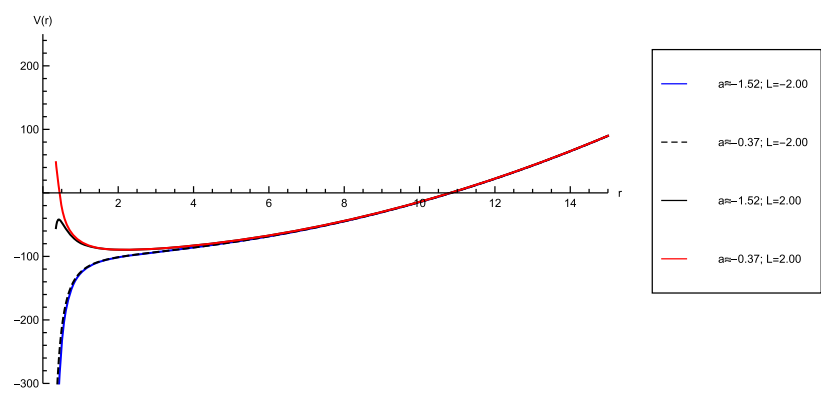

Fig. 5 The behavior of the effective potential $V(r)$ when $r_{u}^{-}=r_{u}^{+}$ for massive particles $m=1$ as a function of $r$ with $\xi=1.2, M=1$, $\lambda=b=1, \Lambda=-1, J=1.2$, and $E=10$. For $L=2, r>0.35$, in order to satisfy the inequality (29), while that for $L=-2$, the inequality (29) is satisfied for all $r$

of the colliding particles. Also, we consider that the particles have the same rest mass, $m_{0}$, energies $E_{1}$ and $E_{2}$ and angular momenta $L_{1}$ and $L_{2}$, respectively. From the relation $E_{\mathrm{CM}}=\sqrt{2} m_{0} \sqrt{1+g_{\mu \nu} u_{1}^{\mu} u_{2}^{v}}$, where $u_{1}$ and $u_{2}$ denote the 3-velocities of the particles, we obtain

$\frac{E_{\mathrm{CM}}^{2}}{2 m_{0}^{2}}=\frac{4 Z^{2} r^{2}\left(r^{2}-L_{1} L_{2}\right)+\left(K_{1} K_{2}-H_{1} H_{2}\right)}{4 Z^{2} r^{4}}$,

where

$K_{i}=2 E_{i} r^{2}-J L_{i}$,

$H_{i}=\sqrt{\left(2 E_{i} r^{2}-J L_{i}\right)^{2}-4 r^{2} Z^{2}\left(r^{2}+L_{i}^{2}\right)}$,

and the subscript takes the values $i=1,2$. Also, when the particles arrive at the event horizon $r=r_{+}, Z^{2}\left(r_{+}\right) \rightarrow 0$, $H_{1} \rightarrow \sqrt{K_{1}^{2}}$ and $H_{2} \rightarrow \sqrt{K_{2}^{2}}$, the CM energy (32) at the horizon is

$\frac{E_{\mathrm{CM}}^{2}}{2 m_{0}^{2}}\left(r \rightarrow r_{+}\right)=\frac{1}{4 Z^{2} r_{+}^{4}}\left(K_{1} K_{2}-\sqrt{K_{1}^{2}} \sqrt{K_{2}^{2}}\right)$.

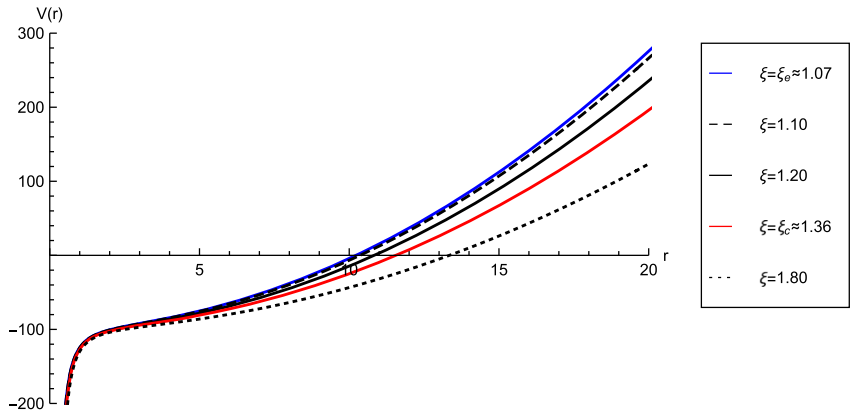

inequality (29) is satisfied, while the right figure shows the behavior of the effective potential for $L=-2$, where inequality (29) is satisfied for all $r$

From the last expression we note that if $K_{1} K_{2}<0$ the $E_{\mathrm{CM}}^{2}$ on the horizon will be a negative infinity; therefore the $\mathrm{CM}$ energy will be imaginary and so it is not a physical solution. In fact, we can set $K_{1}<0$ and $K_{2}>0$ without loss of generality; however, $K_{1}<0$ outside the event horizon is in contradiction with condition (29). However, when $K_{1} K_{2} \geq 0$, the numerator of this expression will be zero and the value of $E_{\mathrm{CM}}$ will be undetermined. Now, in order to find the limiting value of the CM energy at the horizon we can use the L'Hôpital rule, obtaining

$$
\frac{E_{\mathrm{CM}}^{2}}{2 m_{0}}=\frac{r_{+}^{2}\left(K_{1}\left(r_{+}\right)+K_{2}\left(r_{+}\right)\right)^{2}+\left(K_{1}\left(r_{+}\right) L_{2}-K_{2}\left(r_{+}\right) L_{1}\right)^{2}}{2 r_{+}^{2} K_{1}\left(r_{+}\right) K_{2}\left(r_{+}\right)} .
$$

Now, the numerator of the above expression is finite at the horizon and if $K_{i}\left(r_{+}\right)=0$ the CM energy of two colliding particles on the horizon could be arbitrarily high, $\left.E_{\mathrm{CM}}\right|_{K_{i}=0} \rightarrow \infty$. So, from $K_{i}\left(r_{+}\right)=0$ we see that the critical angular momentum is given by

$L_{c i}=\frac{2 r_{+}^{2} E_{i}}{J}, \quad i=1,2$.

On the other hand, when $K_{1}\left(r_{+}\right)$and $K_{2}\left(r_{+}\right)$are both zero, then $E_{\mathrm{CM}}$ is finite at the horizon. In this case $H_{1}\left(r_{+}\right)=$ $H_{2}\left(r_{+}\right)=0$ and

$\frac{E_{\mathrm{CM}}^{2}}{2 m_{0}}=1-\frac{L_{1} L_{2}}{r_{+}^{2}}$.

Therefore, in order to obtain an infinite CM energy only one of the colliding particles must have the critical angular momentum, making the BSW process possible. In Figs. 6 and 7 we show the behavior of $E_{\mathrm{CM}}^{2}\left(r_{+}\right)$versus $L_{1}$ for different values of the Hořava parameter $\xi$ and the aether misalignment parameter $b$, respectively. We observe that there 


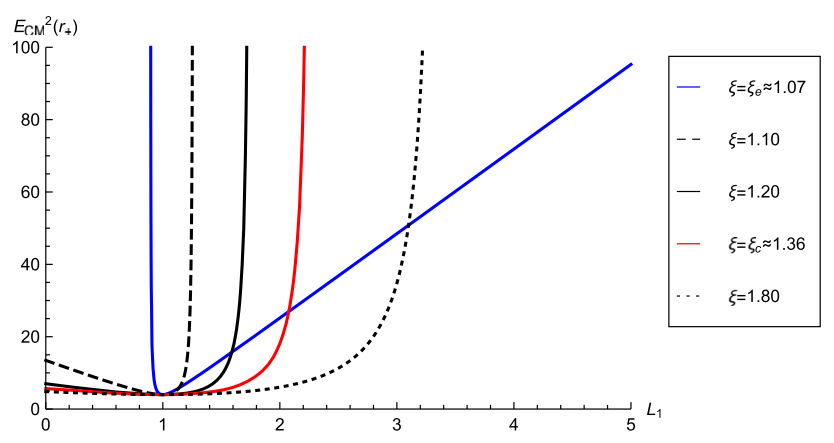

Fig. 6 The behavior of the CM energy $E_{\mathrm{CM}}^{2}\left(r_{+}\right)$at the horizon as a function of $L_{1}$ for different values of $\xi$ with $M=1, \lambda=a=b=1$, $\Lambda=-1$, and $J=1.2$

is a critical value of the angular momentum for particle 1 at which the CM energy blows up. Clearly the figures show that when the parameter $\xi$ increases, $L_{1}=L_{c}$ increases, and the same behavior occurs when the parameter $b$ associated to the aether field increases. Note that only in the extremal case, the limiting value of the critical angular momentum has to be reached from the left of the asymptotic value in order to get a positive CM energy $E_{\mathrm{CM}}^{2}\left(r_{+}\right)$; this indicates that the particles with angular momentum $\left|L_{1}\right|>L_{c}$ start from rest at a finite distance $r_{0}>r_{+}$outside the event horizon and then fall into it. The value of $r_{0}$ will be shown in the next section; in all the other cases it has to be reached from the right. Additionally, in Fig. 8, we have plotted $L_{c}$ in terms of the Hořava parameter $\xi$ for different values of the energy $E$. It is shown that the critical angular momentum $L_{c}$ increases when the energy of particle 1 increases or the Hořava parameter increases.

\section{Radial motion of the particle with critical angular momentum}

Now, we will study the radial motion of the particle with critical angular momentum and energy $E$. As we have mentioned, the particle can reach the event horizon of the black holes if the square of the radial component of the 3-velocity $\dot{r}^{2}$ in Eq. (28) is positive or $V$ is negative in the neighborhood of the black hole horizon. We will denote the explicit form of $\dot{r}^{2}$ with the critical angular momentum by $R^{c}(r)$, which is given by

$R^{c}=\frac{\left(r^{2}-r_{+}^{2}\right)\left(J^{2} m^{2} r^{2}\left(r^{2}-r_{-}^{2}\right) \bar{\Lambda}+E^{2}\left(J^{2}\left(r^{2}-r_{+}^{2}\right)+4\left(r^{2}-r_{-}^{2}\right) r_{+}^{4} \bar{\Lambda}\right)\right)}{J^{2} r^{4}}$,

and it vanishes on the event horizon. Also, for some values of the parameters, $R^{c}$ can be positive, which implies that particles with critical angular momentum can exist outside the event horizon; however, as we shall see, they cannot reach the
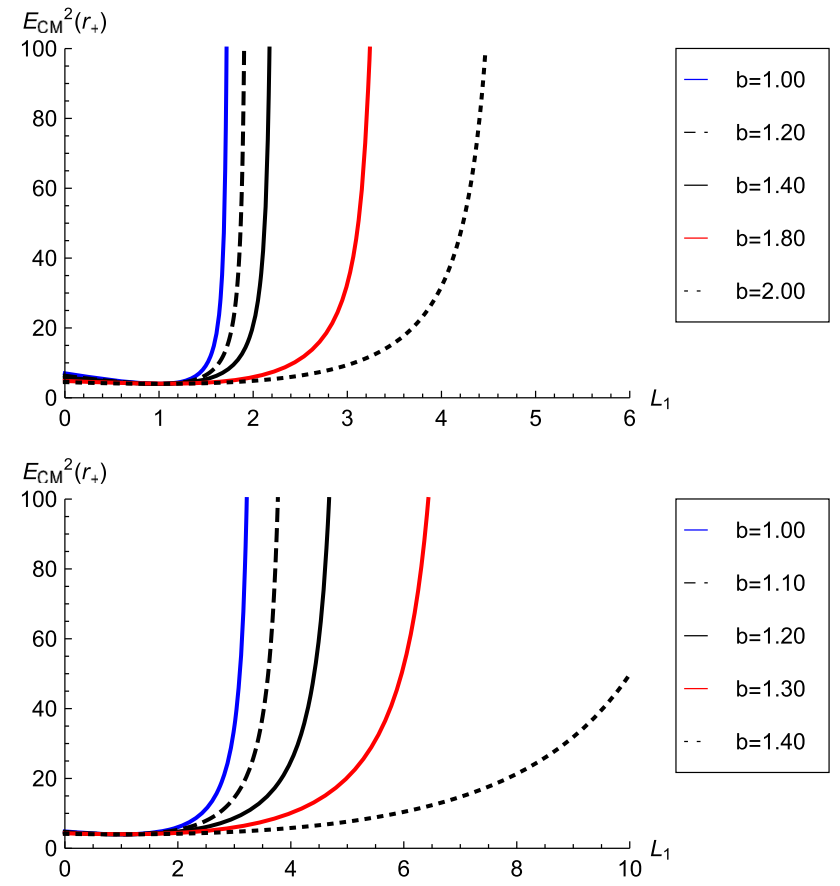

Fig. 7 The behavior of the CM energy $E_{\mathrm{CM}}^{2}\left(r_{+}\right)$at the horizon as a function of $L_{1}$ for $\xi=1.2$ (top panel) and for $\xi=1.8$ (bottom panel) for different values of $b$ with $M=1, \lambda=a=1, \Lambda=-1$, and $J=1.2$

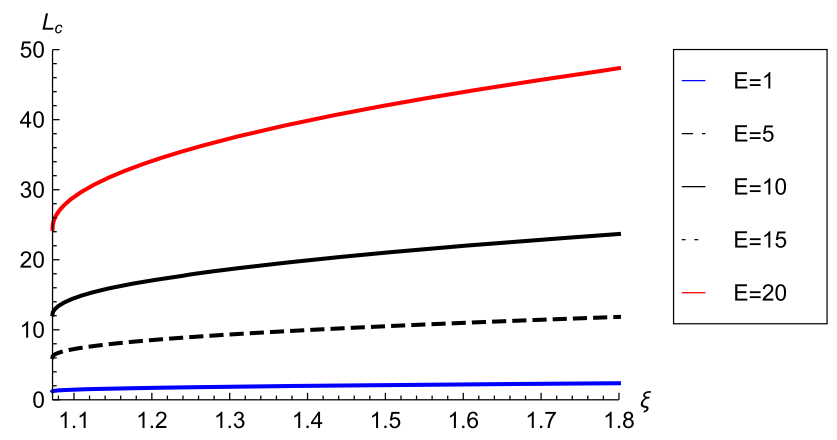

Fig. 8 The behavior of $L_{c}$ as a function of $\xi$ for different values of the energy $E=1,5,10,15$, 20 with $M=1, \lambda=a=b=1, \Lambda=-1$, and $J=1.2$

event horizon unless $r_{+}=r_{-}$. Particles with critical angular momentum can reach the event horizon if the condition $\left.\frac{\mathrm{d} R^{c}}{\mathrm{~d} r}\right|_{r=r_{+}}>0$ is satisfied. From (38) we find

$\left.\frac{\mathrm{d} R^{c}}{\mathrm{~d} r}\right|_{r=r_{+}}=\frac{2 \bar{\Lambda}\left(r_{+}^{2}-r_{-}^{2}\right)}{r_{+}}\left(m^{2}+\frac{4 E^{2} r_{+}^{2}}{J^{2}}\right)<0$,

for $r_{+} \neq r_{-}$; therefore, the massive particle with the critical angular momentum cannot exist just outside of the event horizon $r \gtrsim r_{+}$if the background is AdS. In Fig. 9 we plot the behavior of $R^{c}$ and $\frac{\mathrm{d} R^{c}}{\mathrm{~d} r}$ as a function of $r$ for this background. 
On the other hand, particles with critical angular momentum can reach the Cauchy horizon $r_{-}$if $\left.\frac{\mathrm{d} R^{c}}{\mathrm{~d} r}\right|_{r=r_{-}}>0$. From (38) we find

$$
\left.\frac{\mathrm{d} R^{c}}{\mathrm{~d} r}\right|_{r=r_{-}}=-\frac{2\left(r_{+}^{2}-r_{-}^{2}\right)}{r_{-}}\left(\frac{2 E^{2} r_{+}^{2}}{r_{-}^{4}}+\bar{\Lambda}\left(1+\frac{4 E^{2} r_{+}^{4}}{J^{2} r_{-}^{2}}\right)\right) .
$$

Therefore, the massive particle with critical angular momentum can reach the Cauchy horizon when $-\bar{\Lambda}\left(1+\frac{4 E^{2} r_{+}^{4}}{J^{2} r_{-}^{2}}\right)>$ $\frac{2 E^{2} r_{+}^{2}}{r_{-}^{4}}$ and we have the condition that the two universal horizons coincide to avoid the zone where the aether turns imaginary. For $\bar{J}^{2}<0$ there is no inner horizon $r_{-}$, so the BSW mechanism does not occur.

Furthermore, for the extremal black hole $r_{+}=r_{-}$we obtain

$R^{c}=-\frac{\left(r^{2}-r_{+}^{2}\right)^{2}\left(J^{2} m^{2} r^{2} \bar{\Lambda}+E^{2}\left(J^{2}+4 r_{+}^{4} \bar{\Lambda}\right)\right)}{J^{2} r^{4}}$

and

$$
\begin{aligned}
& \frac{d R^{c}}{d r} \\
& =-\frac{2\left(r^{2}-r_{+}^{2}\right)^{2}\left(J^{2} m^{2} r^{2}\left(r^{2}+r_{+}^{2}\right) \bar{\Lambda}+2 E^{2} r_{+}^{2}\left(J^{2}+4 r_{+}^{4} \bar{\Lambda}\right)\right)}{J^{2} r^{5}} .
\end{aligned}
$$

Then clearly Eqs. (41) and (42) are zero on the event horizon, and it is necessary to calculate $\left.\frac{\mathrm{d}^{2} R^{c}}{\mathrm{~d} r^{2}}\right|_{r=r_{+}}$:

$\left.\frac{\mathrm{d}^{2} R^{c}}{\mathrm{~d} r^{2}}\right|_{r=r_{+}}=8\left(m^{2}|\bar{\Lambda}|-E^{2} \frac{\left(J^{2}-\bar{J}^{2}\right)}{J^{2} r_{+}^{2}}\right)$.

If $\left.\frac{\mathrm{d}^{2} R^{c}}{\mathrm{~d} r^{2}}\right|_{r=r_{+}}>0$, the particle with critical angular momentum will reach the degenerate horizon. This is fulfilled when

$E^{2}<\frac{m^{2}|\bar{\Lambda}| J^{2} r_{+}^{2}}{J^{2}-\bar{J}^{2}}$

Therefore, particles with critical angular momentum satisfying the condition (44) will arrive at the degenerate horizon and thus the BSW process is possible. Note also that, if $\frac{\mathrm{d}^{2} R^{c}}{\mathrm{~d} r^{2}}>0, R^{C}$ has a zero also at $r_{0}=\frac{E \sqrt{J^{2}-4 r_{+}^{4}|\bar{\Lambda}|}}{J m \sqrt{|\bar{\Lambda}|}}$ which is greater than $r_{+}$, and the particles with critical angular momentum can exist at $r_{+} \leq r \leq r_{0}$. In Fig. 9 we plot the behavior of $R^{c}$ and $\frac{\mathrm{d} R^{c}}{\mathrm{~d} r}$ as a function of $r$ for the threedimensional extremal and non-extremal rotating Hořava AdS black hole. We observe that the particle with critical angular momentum can reach the degenerate horizon if the condition (44) is satisfied, and thus the BSW process is possible. It is worth highlighting that this result is different from the usual rotating BTZ black holes ( $\xi=1$ and $\lambda=1$ ), where the massive particles only can exist on the degenerate horizon [46]. Additionally, Fig. 10 shows that for the non-extremal case the region where $\frac{\mathrm{d} R^{c}}{\mathrm{~d} r}>0$ is inside the event horizon $r_{+}$, in particular $\left.\frac{\mathrm{d} R^{c}}{\mathrm{~d} r}\right|_{r=r_{-}}>0$, the massive particles with critical angular momentum can reach the inner horizon $r_{-}$.

\section{Final remarks}

In this paper we considered the collision of two particles in the vicinity of the horizon of a three-dimensional rotating Hořava AdS black hole in the low-energy limit of the theory described by a Lorentz-violating version of the BTZ black hole, i.e. a black hole solution with AdS asymptotics, and we analyzed the energy in the CM frame of the colliding particles in order to investigate the effect of the breaking of Lorentz invariance on the possibility that the black hole can act as a particle accelerator. Thus, the differences observed with respect to the BTZ metric are attributed to the breaking of Lorentz invariance. It is worth mentioning that the results found in this manuscript can be applied for the covariant version of Hořava gravity, where the covariant definition of the center-of-mass energy is well defined.

We showed that, depending on the parameters, the lapse function can represent a spacetime without an event horizon, i.e. a naked singularity, a black hole geometry with one event horizon, an extremal black hole and finally a black hole with two horizons. Also, one of the most interesting features within the context of Lorentz-violating gravity theories is that they can have universal horizons. Thus, it is possible to observe different zones. One of them is limited by $r_{-}$and $r_{+}$, and it is described by the existence of the aether, where the roots of $r_{u}$ are imaginary. Other zones are characterized by two real and distinct universal horizons inside the region between $r_{-}$and $r_{+}$, outside $r_{-}$, and inside $r_{+}$; and we have a particular point where the two universal horizons coincide. In the region between $r_{u}^{-}$and $r_{u}^{+}$, the aether turns imaginary and the foliation cannot be extended until the singularity. So, if this region is located between the inner and outer horizons then the BSW process is not possible on the inner horizon $r_{-}$because the aether is imaginary. We found the following behavior:

- The existence of a massive particle at infinity depends on the effective cosmological constant $(\bar{\Lambda})$ and not on its energy $E$. Thus, massive particles cannot exist at infinity for Hořava AdS black holes in the low-energy limit. 

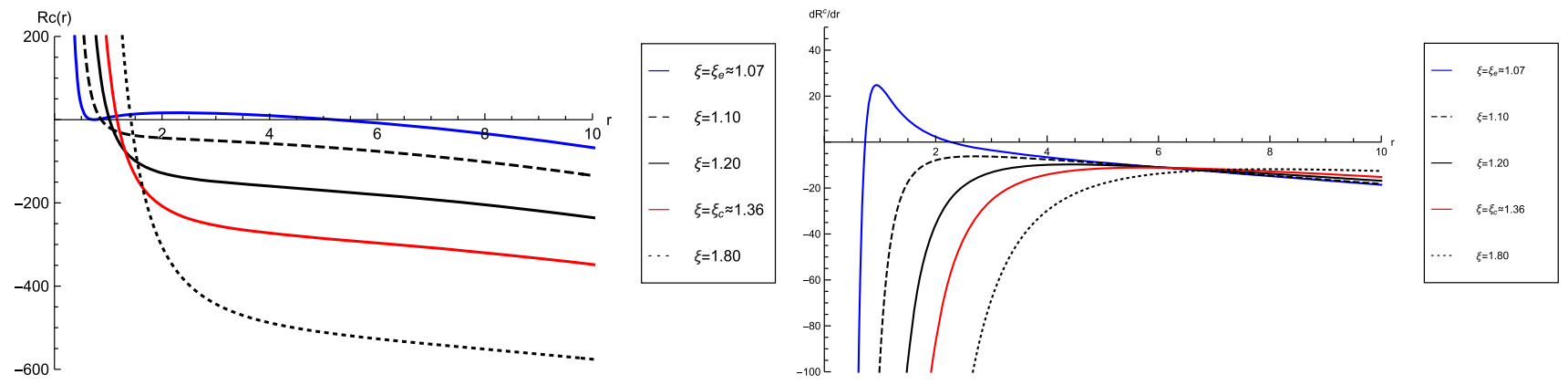

Fig. 9 The behavior of $R^{c}(r)$ (left panel) and $d R^{c}(r) / d r$ (right panel) as a function of $r$ for the extremal (blue line) and non-extremal threedimensional rotating Hořava AdS black hole in the low-energy limit for different values of the parameter $\xi, M=\lambda=a=b=1, \Lambda=-1$, $J=1.2, m=1$ and $E=10$

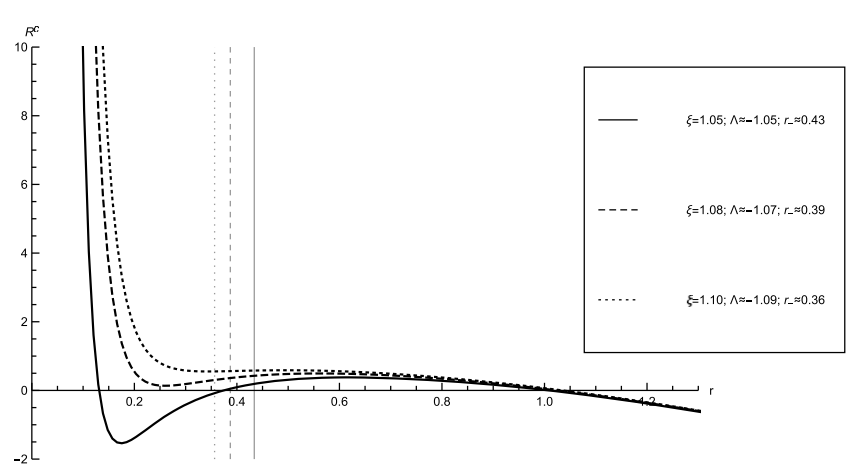

Fig. 10 The behavior of $R^{c}(r)$ (left panel) and $\mathrm{d} R^{c}(r) / \mathrm{d} r$ (right panel) as a function of $r$ for the non-extremal three-dimensional rotating Hořava AdS black hole at the low-energy limit for different values of the parameter $\xi, M=1.2, \lambda=a=b=1, \bar{\Lambda}=-1, J=1$,

- For a background with positive inner and outer horizons $r_{ \pm}, \xi_{e}<\xi<\xi_{c}$, we found that the particles with critical angular momentum will never reach the event horizon; therefore, the black hole cannot act as a particle accelerator with unlimited CM energy on the event horizon. On the other hand, the particles can collide on the inner horizon with arbitrarily high CM energy, possible for the BSW process. Also, we showed that the critical angular momentum increases when the Hořava parameter $\xi$ increases and the aether parameter $b$ increases.

- For the extremal case, $\xi=\xi_{e}$, we found that the particle with critical angular momentum can exist on the degenerate horizon as long as its conserved energy fulfills the condition $E^{2}<\frac{m^{2}|\overline{\mid}| J^{2} r_{+}^{2}}{J^{2}-\bar{J}^{2}}$ with the BSW process being possible. Also, we showed that the critical particle can exist between $r_{+} \leq r \leq r_{0}$, with $r_{0}=\frac{E \sqrt{J^{2}-4 r_{+}^{4}|\bar{\Lambda}|}}{J m \sqrt{|\bar{\Lambda}|}}$. This result is different from the extremal BTZ black holes where particles with critical angular momentum only can exist on the degenerate horizon.

- For $\xi=\xi_{c}$, that is, $r_{-}=0$, the two sectors converge. This occurs when $J^{2}+4 a^{2}(1-\xi)=0(\bar{J}=0)$. Also, the fine

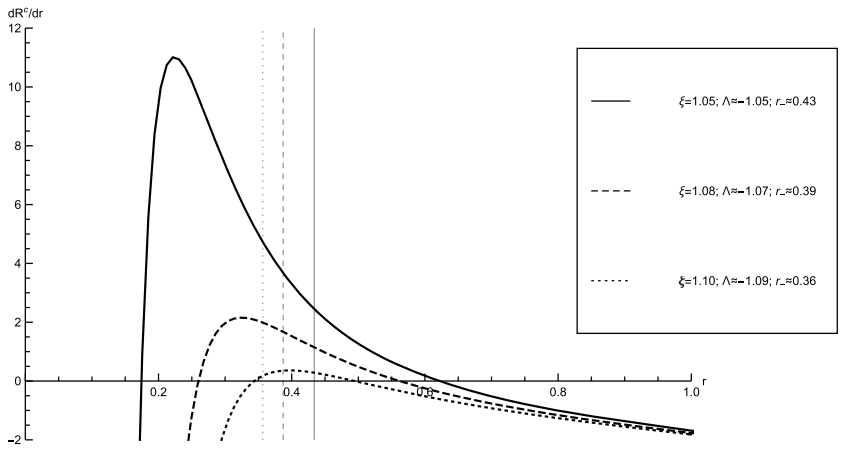

$m=1$ and $E=0.10$. In these cases there are no universal horizons, and the vertical lines correspond to the inner horizon, for each black hole solution

tuning of the critical angular momentum $L_{c}$ is bigger than the extremal and the non-extremal cases, nevertheless the particle with $L_{c}$ will never reach the event horizon.

- In the range $\xi>\xi_{c}$ the black hole only has one horizon $r_{+}>0$. For this case $J^{2}<4 a^{2}(\xi-1)$ and the BSW mechanism is not possible.

Acknowledgements We would like to thank the referee for his/her careful review of the manuscript and his/her valuable comments and suggestions, which helped us to improve the manuscript. Y.V. acknowledge support by the Dirección de Investigación y Desarrollo de la Universidad de La Serena, Grant No. PR18142. P.A.G. would like to thank the Facultad de Ciencias, Universidad de La Serena for its hospitality. Y.V. and R.B. would like to thank the Facultad de Ingeniería y Ciencias, Universidad Diego Portales for its hospitality.

Data Availability Statement This manuscript has no associated data or the data will not be deposited. [Authors' comment: This is a theoretical paper without associated data.]

Open Access This article is licensed under a Creative Commons Attribution 4.0 International License, which permits use, sharing, adaptation, distribution and reproduction in any medium or format, as long as you give appropriate credit to the original author(s) and the source, provide a link to the Creative Commons licence, and indicate if changes were made. The images or other third party material in this article are included in the article's Creative Commons licence, unless indi- 
cated otherwise in a credit line to the material. If material is not included in the article's Creative Commons licence and your intended use is not permitted by statutory regulation or exceeds the permitted use, you will need to obtain permission directly from the copyright holder. To view a copy of this licence, visit http://creativecomm ons.org/licenses/by/4.0/.

Funded by SCOAP ${ }^{3}$.

\section{References}

1. M. Bañados, J. Silk, S.M. West, Kerr black holes as particle accelerators to arbitrarily high energy. Phys. Rev. Lett. 103, 111102 (2009). arXiv:0909.0169 [hep-ph]

2. T. Piran, J. Shaham, J. Katz, Astrophys. J. 196, L107 (1975)

3. T. Piran, J. Shaham, Upper bounds on collisional penrose processes near rotating black hole horizons. Phys. Rev. D 16, 1615 (1977)

4. T. Piran, J. Shaham, Astrophys. J. 214, 268 (1977)

5. A.A. Grib, Y.V. Pavlov, Particles with negative energies in black holes. Int. J. Mod. Phys. D 20, 675 (2011). arXiv: 1008.3657 [gr-qc]

6. O.B. Zaslavskii, Acceleration of particles as universal property of rotating black holes. Phys. Rev. D 82, 083004 (2010). arXiv:1007.3678 [gr-qc]

7. O.B. Zaslavskii, Acceleration of particles by nonrotating charged black holes. JETP Lett. 92, 571 (2010). (Pisma Zh. Eksp. Teor. Fiz. 92, 635 (2010)) arXiv:1007.4598 [gr-qc]

8. S. Gao, C. Zhong, Non-extremal Kerr black holes as particle accelerators. Phys. Rev. D 84, 044006 (2011). arXiv:1106.2852 [gr-qc]

9. Y. Li, J. Yang, Y.L. Li, S.W. Wei, Y.X. Liu, Particle acceleration in Kerr-(anti-) de Sitter black hole backgrounds. Class. Quantum Gravity 28, 225006 (2011). arXiv:1012.0748 [hep-th]

10. J.L. Said, K.Z. Adami, Rotating charged cylindrical black holes as particle accelerators. Phys. Rev. D 83, 104047 (2011). arXiv:1105.2658 [gr-qc]

11. C. Zhong, S. Gao, Particle collisions near the cosmological horizon of a Reissner-Nordstróm de Sitter black hole. JETP Lett. 94, 589 (2011). arXiv:1109.0772 [hep-th]

12. Y. Zhu, S. Fengwu, Y.X. Liu, Y. Jiang, General stationary charged black holes as charged particle accelerators. Phys. Rev. D 84, 043006 (2011)

13. A. Abdujabbarov, N. Dadhich, B. Ahmedov, H. Eshkuvatov, Particle acceleration around a five-dimensional Kerr black hole. Phys. Rev. D 88, 084036 (2013). arXiv:1310.4494 [gr-qc]

14. A. Galajinsky, Near horizon geometry of extremal black holes and Banados-Silk-West effect. Phys. Rev. D 88, 027505 (2013). arXiv:1301.1159 [gr-qc]

15. S.R. Shaymatov, B.J. Ahmedov, A.A. Abdujabbarov, Particle acceleration near a rotating black hole in a Randall-Sundrum brane with a cosmological constant. Phys. Rev. D 88(2), 024016 (2013)

16. J. Sadeghi, B. Pourhassan, H. Farahani, Rotating charged hairy black hole in $(2+1)$ dimensions and particle acceleration. Commun. Theor. Phys. 62(3), 258 (2014). arXiv:1310.7142 [hep-th]

17. S. Fernando, String black hole: can it be a particle accelerator? Gen. Relativ. Gravit. 46, 1634 (2014). arXiv:1311.1455 [gr-qc]

18. S.G. Ghosh, P. Sheoran, M. Amir, Rotating Ayón-Beato-García black hole as a particle accelerator. Phys. Rev. D 90(10), 103006 (2014). arXiv:1410.5588 [gr-qc]

19. P. Pradhan, Charged dilation black holes as particle accelerators. Astropart. Phys. 62, 217 (2015). arXiv:1407.0877 [gr-qc]

20. S.G. Ghosh, M. Amir, Horizon structure of rotating Bardeen black hole and particle acceleration. Eur. Phys. J. C 75(11), 553 (2015). arXiv:1506.04382 [gr-qc]

21. M. Amir, S.G. Ghosh, Rotating Hayward's regular black hole as particle accelerator. JHEP 1507, 015 (2015). arXiv:1503.08553 [gr$\mathrm{qc}]$
22. M. Guo, S. Gao, Kerr black holes as accelerators of spinning test particles. Phys. Rev. D 93(8), 084025 (2016). arXiv:1602.08679 [gr-qc]

23. Y.P. Zhang, B.M. Gu, S.W. Wei, J. Yang, Y.X. Liu, Charged spinning black holes as accelerators of spinning particles. Phys. Rev. D 94(12), 124017 (2016). arXiv:1608.08705 [gr-qc]

24. C. Armaza, M. Bañados, B. Koch, Collisions of spinning massive particles in a Schwarzschild background. Class. Quantum Gravity 33(10), 105014 (2016). arXiv:1510.01223 [gr-qc]

25. O.B. Zaslavskii, Schwarzschild black hole as particle accelerator of spinning particles. EPL 114(3), 30003 (2016). arXiv:1603.09353 [gr-qc]

26. O.B. Zaslavskii, Black hole with a scalar field as a particle accelerator. Int. J. Mod. Phys. D 26(10), 1750108 (2017). arXiv:1602.08779 [gr-qc]

27. S. Fernando, Spinning dilaton black hole in $2+1$ dimensions as a particle accelerator. Mod. Phys. Lett. A 32, 1750074 (2017). arXiv: 1703.00373 [gr-qc]

28. P.A. González, M. Olivares, E. Papantonopoulos, Y. Vásquez, Motion and collision of particles in a rotating linear dilaton black hole. Phys. Rev. D 97(6), 064034 (2018). arXiv:1802.01760 [gr-qc]

29. P.A. González, M. Olivares, Y. Vásquez, J. Saavedra, A. Övgün, Motion and collision of particles near DST Black holes. Eur. Phys. J. C 79(6), 528 (2019). arXiv:1811.08551 [gr-qc]

30. J. Jiang, S. Gao, Universality of BSW mechanism for spinning particles. Eur. Phys. J. C 79(5), 378 (2019). arXiv:1905.02491 [hepth]

31. F. Atamurotov, B. Ahmedov, S. Shaymatov, Formation of black holes through BSW effect and black hole-black hole collisions. Astrophys. Space. Sci. 347, 277 (2013)

32. P. Horava, Quantum gravity at a Lifshitz point. Phys. Rev. D 79, 084008 (2009). arXiv:0901.3775 [hep-th]

33. T. Jacobson, D. Mattingly, Phys. Rev. D 64, 024028 (2001). arXiv:gr-qc/0007031

34. T.P. Sotiriou, M. Visser, S. Weinfurtner, Lower-dimensional Horava-Lifshitz gravity. Phys. Rev. D 83, 124021 (2011). arXiv:1103.3013 [hep-th]

35. T.P. Sotiriou, I. Vega, D. Vernieri, Rotating black holes in threedimensional Hořava gravity. Phys. Rev. D 90(4), 044046 (2014). arXiv: 1405.3715 [gr-qc]

36. R. Bécar, P.A. González, E. Papantonopoulos, Y. Vásquez, Quasinormal modes of three-dimensional rotating Hořava AdS black hole and the approach to thermal equilibrium. Eur. Phys. J. C 80(7), 600 (2020). arXiv: 1906.06654 [gr-qc]

37. P.A. González, M. Olivares, E. Papantonopoulos, Y. Vásquez, Motion and trajectories of photons in a three-dimensional rotating Hořava-AdS black hole. Phys. Rev. D 101(4), 044018 (2020). arXiv: 1912.00946 [gr-qc]

38. P.A. González, M. Olivares, E. Papantonopoulos, Y. Vásquez, Time like geodesics in three-dimensional rotating Hořava AdS black hole. arXiv:2008.00933 [gr-qc]

39. T. Zhu, Q. Wu, M. Jamil, K. Jusufi, Shadows and deflection angle of charged and slowly rotating black holes in Einstein-Æther theory. Phys. Rev. D 100(4), 044055 (2019)

40. A. Abdujabbarov, B. Ahmedov, B. Ahmedov, Energy extraction and particle acceleration around rotating black hole in HoravaLifshitz gravity. Phys. Rev. D 84, 044044 (2011). arXiv:1107.5389 [astro-ph.SR]

41. J. Sadeghi, B. Pourhassan, Particle acceleration in Horava-Lifshitz black holes. Eur. Phys. J. C 72, 1984 (2012). arXiv:1108.4530 [hepth]

42. M. Halilsoy, A. Ovgun, Particle collision near $1+1$-dimensional Horava-Lifshitz black hole and naked singularity. Adv. High Energy Phys. 2017, 4383617 (2017). arXiv:1504.03840 [gr-qc]

43. M. Azreg-Aïnou, Z. Chen, B. Deng, M. Jamil, T. Zhu, Q. Wu, Y.K. Lim, Orbital mechanics and quasiperiodic oscillation resonances of 
black holes in Einstein-Æther theory. Phys. Rev. D 102(4), 044028 (2020). arXiv:2004.02602 [gr-qc]

44. J. Rayimbaev, A. Abdujabbarov, M. Jamil, W. Han, Dynamics of magnetized particles around Einstein-Æther black hole with uniform magnetic field. arXiv:2009.04898 [gr-qc]

45. K. Lake, Particle accelerators inside spinning black holes. Phys. Rev. Lett. 104, 211102 (2010). (Erratum: (Phys. Rev. Lett. 104, 259903 (2010))) arXiv:1001.5463 [gr-qc]

46. J. Yang, Y.L. Li, Y. Li, S.W. Wei, Y.X. Liu, Particle collisions in the lower dimensional rotating black hole space-time with the cosmological constant. Adv. High Energy Phys. 2014, 204016 (2014). arXiv: 1202.4159 [hep-th]

47. N. Tsukamoto, K. Ogasawara, Y. Gong, Particle collision with an arbitrarily high center-of-mass energy near a BañadosTeitelboim-Zanelli black hole. Phys. Rev. D 96(2), 024042 (2017). arXiv: 1705.10477 [gr-qc]
48. R. Bécar, P.A. González, Y. Vásquez, Particle collisions near a three-dimensional warped AdS black hole. Eur. Phys. J. C 78(4), 335 (2018). arXiv:1712.00868 [gr-qc]

49. B. Cropp, S. Liberati, A. Mohd, M. Visser, Ray tracing EinsteinÆther black holes: Universal versus Killing horizons. Phys. Rev. D 89(6), 064061 (2014). arXiv:1312.0405 [gr-qc]

50. S. Chandrasekhar, The Mathematical Theory of Black Holes (Oxford University Press, New York, 1983) 